\title{
IMPROVING SOLUBILITY OF BCS CLASS II DRUGS USING SOLID DISPERSION: A REVIEW
}

\author{
Sweta Mehta $^{1}$, Nisha Mary Joseph ${ }^{1}$, Fitsum F. Sahle ${ }^{1}$, S. Palani ${ }^{2}$ \\ 1. Department of Pharmaceutics and Social Pharmacy, Addis Ababa University, Addis Ababa, Ethiopia \\ 2. College of Health Sciences, Jijiga University, Jijiga, Ethiopia
}

\begin{abstract}
Biopharmaceutics Classification System is important for determining the bioavailability of the drugs. Drug development tool that allows estimation of the three major factors that affect oral drug absorption from immediate release solid oral dosage form is Dissolution, Solubility and Intestinal permeability. The bioavailability issue can be due to insufficient solubility of permeability. Most compounds face the solubility problems. The dissolution rate of drug from its dosage form is considered as an important parameter in the absorption. Dissolution is the rate-limiting step in the absorption of drugs from one solid dosage form especially when the drug is poorly water soluble. Poor wettability of drugs leads to the decrease in their bioavailability. Presently only $8 \%$ of new drug candidates have both high solubility and permeability and more than $60 \%$ of the products have poor water solubility. As a result the potentially important drugs do not reach the market and are not achieving their full potential. Hence, with the advancement of chemical science, the need of development of pharmaceutical technologies is also increasing. Solid dispersions have attracted considerable interest as an efficient means of improving the dissolution rate and hence the bioavailability of many drugs belonging to BCS class II. Solid dispersion has been used over last 20 to 30 years and has been known to give fruitful result in improving the release rate and oral bioavailability of poorly water soluble drugs. Solid dispersion with different polymers and at different ratios is carried out and is known to show good release when compared to the drug alone and the physical mixture. Improvement in dissolution of drug was observed in all the solid dispersions as compared to pure drug.

Keywords: Solid dispersion,eutectic mixtures,isothermal titration calorimetry,kneading method
\end{abstract}

\section{INTRODUCTION}

Oral route is the most preferred route of administration of the drug due to various reasons like its convenience, good patient acceptance and low medicine production $\operatorname{costs}^{1}$. The solubility and permeability of the drug is a key determinant for its oral bioavailability. There have always been certain drugs for which solubility has presented a challenge for developing suitable formulation for oral administration ${ }^{2}$. Studies have shown that $40 \%$ of the new chemical entities are poorly soluble in water of insoluble in water and upto $50 \%$ of the orally administered drug compound suffer from formulation problem related to their low solubility and high lipophilicity. For a drug to be absorbed in the systemic circulation after the oral administration need to be dissolved in the gastric fluid ${ }^{1}$. However, a poorly water soluble drug with poor solubility and low dissolution rate in the aqueous gastrointestinal fluid is a reason for its insufficient bioavailability. Solubility of such drugs can be improved by incorporating the drug in a hydrophilic carrier material obtaining a product called solid dispersion $^{3}$.

In 1961, Sekiguchi and obi first proposed the utilization of solid dispersion inorder to increase the dissolution and oral absorption of poorly water soluble drugs ${ }^{4}$. In 1971 Chiou and Riegelman defined Solid dispersion as "dispersion of one or more active ingredients in an inert (c) 2011-14, JDDT. All Rights Reserved carrier or matrix at solid state prepared by the melting (fusion), solvent, or melting solvent method" 5 . The solid dispersion may also be called solid state dispersion, as first used by Mayerson and Gibaldi ${ }^{6}$. Solid dispersion has been used as an effective method to improve the bioavailability and dissolution rate of poorly water soluble drugs ${ }^{7}$.

In the Biopharmaceutical Classification System the class II drugs show low aqueous solubility and high membrane permeability. Thus, Solid dispersion technologies give promising results for improving the oral absorption and bioavailability of the drugs belonging to BCS class II ${ }^{8}$. Though salt formation, particle size reduction, solubilization ${ }^{9}$, and complexation with cyclodextrin ${ }^{8}$ have been used to increase the dissolution rate and thereby the oral absorption and bioavailability of poorly water soluble drugs ${ }^{10}$. However these techniques have limitations. On the other hand, solid dispersion formulations offer various processing and excipients an option that allows more flexibility for formulating oral delivery system for poorly water soluble drugs ${ }^{8}$.

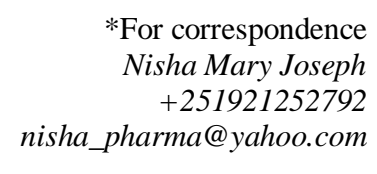

ISSN: 2250-1177

CODEN (USA): JDDTAO 
The solubility and bioavailability of poorly water soluble drug can be improved by converting the drug into amorphous state ${ }^{11}$. The mechanism by which the solid dispersion enhances the solubility and dissolution involves particle size reduction to fine molecular level, conversion of crystalline form to amorphous form and by enhancing wettability ${ }^{12}$.

\section{ADVANTAGE OF SOLID DISPERSION \\ i) Particles with reduced particle size}

Molecular dispersion represents the last stage of particle size reduction, solid dispersion uses this principle where the drug is molecularly dispersed in the dissolution medium after the dissolution of the carrier $^{8}$. As a result high surface area is formed and thus increased dissolution and consequently improved bioavailability of poorly water soluble $\operatorname{drug}^{13}$.

\section{ii) Particles with improved wettability}

Drug solubility enhancement is related to the drug wettability improvement ${ }^{13}$. The use of carriers without surface activity (eg. Urea) and with surface activity (eg. Cholic acid and bile salt) improved drug wettability. Moreover, carriers improve the drug dissolution profile by direct dissolution or co-solvent effect $^{8}$.

\section{iii) Particles with high porosity}

The dissolution rate of the solid dispersion increases when carriers with high degree of porosity are used. For example solid dispersion containing linear polymer have larger and more porous particles and thus gives higher dissolution rate ${ }^{13}$.

\section{iv) Particles in amorphous state}

Drugs in amorphous state tend to have higher solubility then the drugs in crystalline state and it requires energy to break the crystal lattice. In solid dispersion the drug exists as supersaturated solution after system dissolution and it is considered when drug precipitate, it exists as a metastable polymorphic form with higher dissolution then the crystal form ${ }^{8}$.

\section{DISADVANTAGE OF SOLID DISPERSION}

The major disadvantage of solid dispersion is related to the instability ${ }^{13}$. During storage the effect of moisture on the stability of amorphous pharmaceuticals is of significant concern, as it increases the drug mobility and promotes drug crystallization. An obstacle in solid dispersion method in pharmaceutical development is the amount of carrier needed i.e. more than $50 \%$ to $80 \%$ is required to achieve desired dissolution. In addition the polymer used for solid dispersion also absorbs moisture which may result in phase separation, crystal growth or conversion to the crystalline form. Thus results in decreased solubility and dissolution rate ${ }^{14}$.

\section{Limitations of Solid Dispersion}

i) The physical and chemical stability of drugs and vehicles,

ii) Method of preparation,

iii) Reproducibility of its physicochemical properties, ${ }^{14}$

iv) Formulation of solid dispersion into dosage forms, and

v) Scale-up of manufacturing processes ${ }^{13}$.

vi)

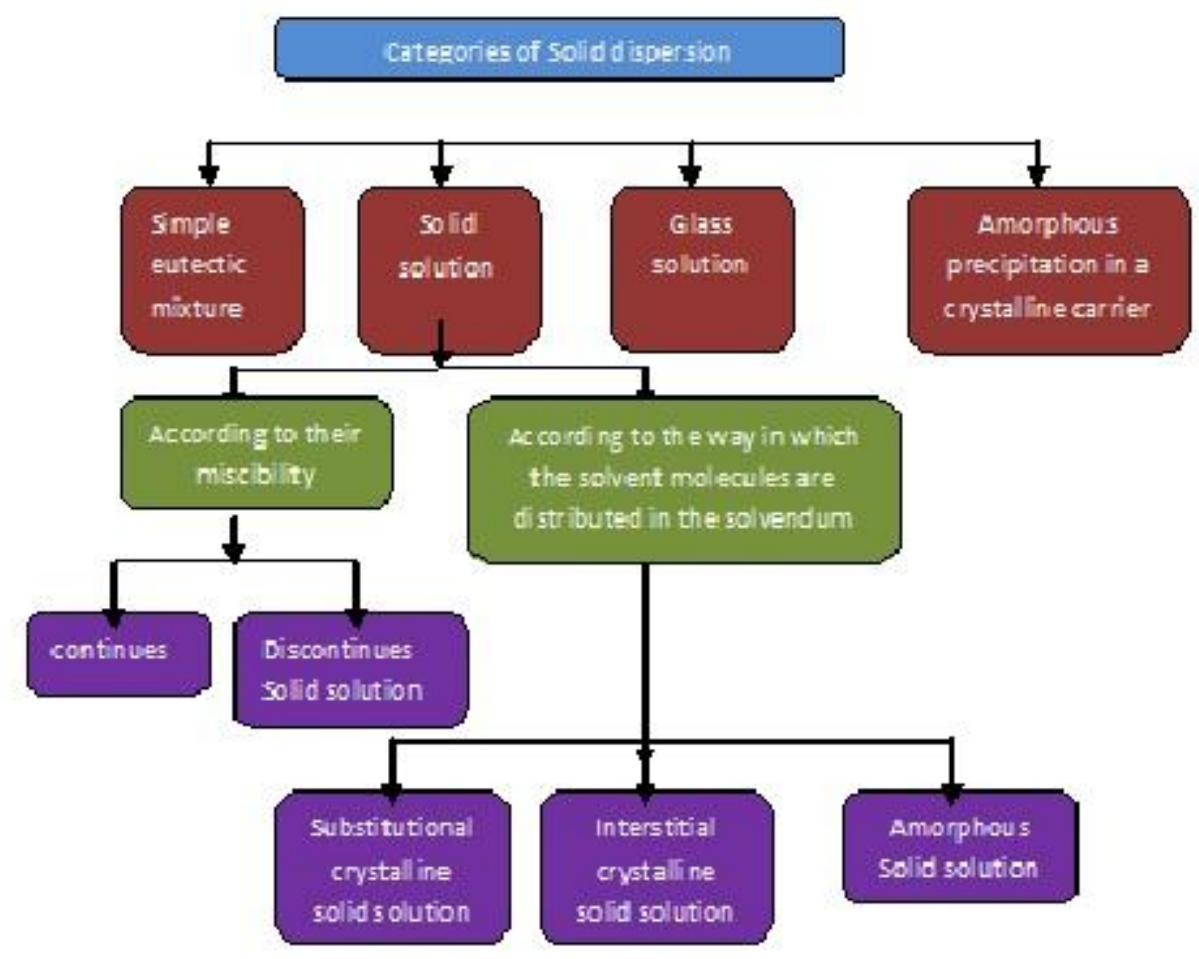

Figure 1: Categories of Solid Dispersion ${ }^{16}$ 


\section{TYPES OF SOLID DISPERSION}

a) Eutectic mixtures: It consists of two completely miscible compounds in liquid state. However it has very limited miscibility in solid state. It is prepared by rapid solidification of fused melt of two components which has complete liquid miscibility.

b) Solid solution: It consists of just one phase irrespective of the number of components. In solid solution the particle size of the drug has been reduced to absolute minimum and the dissolution rate is determined by the dissolution rate of the carrier. c) Glass solution and suspensions: Glass solutions are homogeneous glassy system in which solute dissolves in glass carrier. Glass suspensions are mixture in which precipitated particles are suspended in glass solvent. Lattice energy is much lower in glass solution and suspension.

d) Amorphous precpitation in crystalline matrix: This is similar to simple eutectic mixtures. However it differs from simple eutectic mixture by that the drug is precipitated out in amorphous form ${ }^{15}$.

Table 1: Types of solid dispersion (Chiou and Riegelman Classification) ${ }^{17}$

\begin{tabular}{|c|c|c|c|c|c|}
\hline S.No. & $\begin{array}{l}\text { Solid dispersion } \\
\text { type }\end{array}$ & Matrix & Drug & Observation & $\begin{array}{l}\text { No. of } \\
\text { Phases }\end{array}$ \\
\hline 1. & Eutectics & $\begin{array}{l}\text { Crystalline } \\
\text { stare }\end{array}$ & $\begin{array}{l}\text { Drug dispersed as crystalline } \\
\text { particles in the matrix }\end{array}$ & $\begin{array}{l}\text { The first type of } \\
\text { dispersion prepared }\end{array}$ & 2 \\
\hline 2. & $\begin{array}{l}\text { Amorphous } \\
\text { precipitation in } \\
\text { crystalline matrix }\end{array}$ & $\begin{array}{l}\text { Crystalline } \\
\text { state }\end{array}$ & $\begin{array}{l}\text { A drug dispersed } \text { as } \\
\text { amorphous clusters in the } \\
\text { matrix }\end{array}$ & Rarely encountered & 2 \\
\hline \multirow[t]{5}{*}{3.} & Solid solution & - & - & - & - \\
\hline & $\begin{array}{l}\text { Continuous solid } \\
\text { solution }\end{array}$ & $\begin{array}{l}\text { Crystalline } \\
\text { state }\end{array}$ & $\begin{array}{l}\text { Drug molecularly dispersed } \\
\text { throughout the matrix }\end{array}$ & $\begin{array}{l}\text { Miscible at all composition, } \\
\text { never prepared }\end{array}$ & 1 \\
\hline & $\begin{array}{l}\text { Discontinuous } \\
\text { solid solution }\end{array}$ & $\begin{array}{l}\text { Crystalline } \\
\text { state }\end{array}$ & $\begin{array}{l}\text { Drug molecularly dispersed } \\
\text { throughout the matrix }\end{array}$ & $\begin{array}{l}\text { Partially miscible, } 2 \text { phases } \\
\text { even though drug is } \\
\text { molecularly dispersed }\end{array}$ & 2 \\
\hline & $\begin{array}{l}\text { Substitutional solid } \\
\text { solution }\end{array}$ & $\begin{array}{l}\text { Crystalline } \\
\text { state }\end{array}$ & $\begin{array}{l}\text { Drug molecularly dispersed } \\
\text { throughout the matrix }\end{array}$ & $\begin{array}{l}\text { Molecular diameter of drug } \\
\text { (solute) differ less than } 15 \% \\
\text { from the matrix (solvent) } \\
\text { diameter }\end{array}$ & 1 or 2 \\
\hline & $\begin{array}{l}\text { Interstitial solid } \\
\text { solution }\end{array}$ & $\begin{array}{l}\text { Crystalline } \\
\text { state }\end{array}$ & $\begin{array}{l}\text { Drug molecularly dispersed } \\
\text { throughout the matrix }\end{array}$ & $\begin{array}{l}\text { Drug molecular diameter less } \\
\text { than } 59 \% \text { of matrix diameter. } \\
\text { Usually limited miscibility, } \\
\text { Discontinuous. Example: Drug } \\
\text { in helical interstitial space of } \\
\text { PEG }\end{array}$ & 2 \\
\hline 4. & Glass suspension & $\begin{array}{l}\text { Amorphous } \\
\text { state }\end{array}$ & 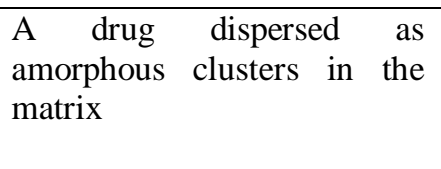 & $\begin{array}{l}\text { Particle size of dispersed phase } \\
\text { dependent on cooling/ } \\
\text { evaporation rate many solid } \\
\text { dispersion are of this type }\end{array}$ & 2 \\
\hline 5. & Glass Solution & $\begin{array}{l}\text { Amorphous } \\
\text { state }\end{array}$ & $\begin{array}{l}\text { Drug molecularly dispersed } \\
\text { throughout the matrix }\end{array}$ & $\begin{array}{l}\text { Requires miscibility/ solid } \\
\text { solubility or complex formation } \\
\text { on fast cooling or evaporation } \\
\text { during preparation, many } \\
\text { example especially with PVP }\end{array}$ & 1 \\
\hline
\end{tabular}




\section{METHODS OF SOLID DISPERSION}

a) Fusion method: it is also known as melt method when the starting materials used are crystalline ${ }^{13}$. Sekiguchi et al. in 1961 were the first to use melting method which involved melting the drug within the carrier followed by cooling and pulverization of the obtained product ${ }^{17}$. Ex. Albendazole and urea solid dispersion was prepared by this method ${ }^{18}$. The main advantages of this method are its simplicity and economy. is However it has few limitations like it is applied only when the drug and the matrix are compatible and when they mix well at the heating temperature. Phase separation may also occur during cooling, the drug matrix miscibility changes ${ }^{13}$.

b) Solvent Evaporation method: the drug and the carrier are completely dissolved in an organic solvent, the solvent is evaporated. The obtained solid mass is ground, sieved and dried. Ex. Solid dispersion of ofloxacin with PEG by solvent evaporation method ${ }^{16}$.

c) Modified Solvent Evaporation method: drug is dissolved in organic solvent at its saturation solubility with continuous stirring for some time. The polymer is suspended in sufficient amount of water. The drug solution is poured at once into polymer suspension. The entire solvent is evaporated. The mass obtained is dried ${ }^{16}$.

d) Melting Solvent method: it is a combination of two methods which involves dissolving the drug in a suitable solvent and mixing it with molten carrier followed by cooling ${ }^{15}$. The solidified mass is crushed, pulverized and passed through sieve. The advantage of this method is it reduces maximum temperature and results in less decomposition of thermolabile drugs.

e) Kneading method: A mixture of accurately weighed drug and carrier is wetted with solvent and kneaded thoroughly for some time in a glass mortar. The paste formed is dried and sieved. Ex. furosemide and crospovidone solid dispersion was prepared by this method ${ }^{19}$.

f) Co-grinding: the drug and carriers physical mixture is mixed in a blender at a particular speed. It is then charged into a chamber of vibration ball mill. The powder mixture is pulverized and collected at room temperature. Ex chlordiazepoxide and mannitol solid dispersion was prepared by this method ${ }^{18}$.

g) Co-precipitation method: the carrier is dissolved in water and drug in organic solvent, then the aqueous solution of carried is poured into organic solution of drug. During the addition of non-solvent the drug and carrier are co-precipitated to form micro particles. The resultant micro particle suspension is filtered and dried (solid dispersion) ${ }^{20}$.

h) Spray drying method: here the solution of drug substance and carrier is evaporated by spraying the solution as fine droplets in chamber with maintained temperature, humidity and airflow. The principle involved is evaporation of the solvent from the mixture until the desired moisture content is obtained. It is advantageous for weight and volume reduction ${ }^{15}$. Rankell et al. prepared solid dispersion of Loperamide with PEG $6000^{21}$.

i) Direct capsule filling: filling of semisolid material in to and hard gelatin capsule as melt, this method was first described by Francois and Jones ${ }^{15}$. The molten dispersion forms a solid plug inside the capsule on cooling at room temperature. This method is advantageous as it reduces cross contamination, exposure in dust free environment, better filling and content uniformity can be obtained $^{20}$

j) Lyophillization technique: it has been thought of a molecular mixing technique where the drug and carrier are co dissolved in a common solvent, frozen and sublimed to obtain a lyophilized molecular dispersion ${ }^{21}$.

k) Gel entrapment technique: polymer like HPMC is dissolved in an organic solvent until clear and transparent gel is obtained. The drug is then added to the gel and sonicated for few minutes. The organic solvent is evaporated the solid dispersion obtained is reduced in size and sieved ${ }^{22}$.

\section{CARRIERS FOR SOLID DISPERSION}

Carriers play an important role in influencing the dissolution characteristic of dispersed drug. The carrier needs to meet the following requirement.

Freely soluble in water and with rapid dissolution property.

Soluble in wide variety of solvents

Chemically compatible with the drug

Heat stable with low melting point for melt method. ${ }^{23}$

Non toxic and pharmacologically inert ${ }^{24}$.

First generation carriers: Crystalline carriers: Urea, Sugars, and Organic acids. They have disadvantage of forming crystalline solid dispersion that is thermodynamically more stable and do not release the drug quickly ${ }^{24}$.

Second generation carriers: Amorphous carriers: Polyethylene glycol, Povidone, Polyvinylacetate, Polymethacrylate, cellulose derivatives ${ }^{25}$.

Third generation carriers: Surface active self emulsifying carriers: Poloxamer 408, Tween 80, Gelucire 44/14. These carriers have been effective in originating high polymorphic purity and enhanced in vivo bioavailability ${ }^{25}$.

\section{CHARACTERIZATION OF SOLID DISPERSION}

a) Physical characterization and saturation solubility

An excess amount of the physical mixture and solid dispersion is taken in a conical flask and $10 \mathrm{ml}$ of distilled water is added and shaken on rotator shaker for 48 hour at $37^{\circ} \mathrm{C}$. Remove the flask and filter it. Suitable amount of aliquot is withdrawn from the filtered solution and the drug content was analyzed after appropriate dilution with distilled water and was compared with pure drug solubility ${ }^{26}$.

b) Fourier Transform Infrared Spectroscopic Analysis 
The interaction between the drug and the carrier in solid dispersion is assessed by FTIR. In the solid dispersion preparation the peak band shows shift in the absorption spectrum for the drug molecule. However, Careful interpretation of the spectrum is required for some of the delicate changes ${ }^{27}$

c) Differential Scanning Calorimetry

The calibration of the DSC temperature and enthalpy scale was carried out using Indium/Zinc standards. The sample are taken in an aluminum pan and is hermetically sealed and heated at a constant rate of $20^{\circ} \mathrm{C}$ per minute over a temperature range of 20 to $150^{\circ} \mathrm{C}$. The inert atmosphere was maintained by purging nitrogen gas at flow rate of $50 \mathrm{ml} / \mathrm{min}^{28}$.

d) Hyper Differential Scanning Calorimetry (Hyper DSC)

This method employs excess heating rate starting from 100 to $500^{\circ} \mathrm{C} / \mathrm{min}$. However, in DSC the heating rate ranges from about 1 to $20^{\circ} \mathrm{C} / \mathrm{min}$. Hyper differential scanning calorimetry have number of advantages, which involves its use to prevent recrystalisation during the heating step. Hyper DSC when compared to the standard DSC thermograms can also be used to enhance thermal signals it also have advantage of minimizing and preventing the changes in morphology and interaction during the heating process ${ }^{29}$

e) X Ray Powder Diffraction (XRPD)

To characterize the physical form of the drug in the sample $X$ ray diffraction study is carried out. In this method the sample is spread on a glass slide with an appropriate thickness of $0.5 \mathrm{~mm}$. The slide is then placed in X-ray diffractometer vertically at $0^{\circ}$ angle so that the X-ray beams falls on it. The results were then recorded over an range of 0 to $90^{\circ} \mathrm{C}^{30}$.

f) Scanning electron microscopy

In this method both the pure drug and the solid dispersion sample and mounted on the stub using double sided adhesive tape and coated with gold palladium alloy. The samples are then analyzed using scanning electron microscope for external morphology ${ }^{31}$.

g) In Vitro release Studies

The dissolution a study is carried out using accurately weighed amount of sample. The sample is withdrawn at predetermined time interval and same amount of aliquots is replaced by fresh dissolution medium. The drug release was then analyzed UV spectrophotometrically or HPLC ${ }^{32}$.

h) Isothermal titration calorimetry (ITC)

It is important method for understanding biological processes at molecular level. In this method a small change of heat during the reaction can be detected. This method is also useful in determining thermodynamic parameters like Gibbs free energy, enthalpy, entropy, binding constant, heat capacity and effective numbers of binding site in the biological reaction. This method has advantages like it is the only technique that can establish all binding site in the biological reaction. It also allows the measurement of heat signals ${ }^{29}$.

Several Marketed and late stage drugs are designed for improved solubility by solid dispersion

\begin{tabular}{|c|c|c|c|}
\hline Product/Substance & $\begin{array}{c}\text { Dispersion Polymer or } \\
\text { Carrier }\end{array}$ & Technology used & Company \\
\hline Gris-PEG ${ }^{\infty}$ (Griseofulvin) & Polyethylene glycol & $\begin{array}{l}\text { Melt process; exact } \\
\text { process unknown }\end{array}$ & Novartis \\
\hline $\begin{array}{l}\text { Sproramax capsules } \\
\text { (Itraconazole) }\end{array}$ & $\begin{array}{l}\text { Hydroxypropylmethylcellulose } \\
\text { (HPMC) }\end{array}$ & Spray layering & $\begin{array}{l}\text { Janseen } \\
\text { pharmaceutica }\end{array}$ \\
\hline Cesamet $^{\circledR}$ ( Nabilone) & Providone & process unknown & Lilly \\
\hline $\begin{array}{l}\text { Kaletra (lopinavir and } \\
\text { ritonavir) }\end{array}$ & $\begin{array}{l}\text { Polyvinylpyrolidone } \\
\text { (PVP)/polyvinyl acetate }\end{array}$ & Melt-extrusion & $\begin{array}{l}\text { Abbott } \\
\text { Laboratories }\end{array}$ \\
\hline Torcetrapib $^{a}$ & HPMC acetate succinate & Spray drying & Pfizer \\
\hline Ibuprofen & Various & Melt-extrusion & Soliqs \\
\hline Isopt in SRE-240 (Verapamil) & Various & Melt-extrusion & Soliqs \\
\hline Rezulin ${ }^{\mathrm{b}}$ (Troglitazone) & PVP & Melt-extrusion & Pfizer \\
\hline LCP-Tacro (Tracrolimus) & HPMC & Melt-granulation & Life Cycle Pharma \\
\hline Intelence (Etravirine) & HPMC & Spray drying & Tibotec \\
\hline Certican (Everolimus) & HPMC & Melt or Spray drying & Novartis \\
\hline Afeditab (Nifedipine) & Poloxomer or PVP & Melt/absorb on carrier & Élan Corp. \\
\hline
\end{tabular}

a Halted in phase III; b Withdrawn from market. 


\section{CURRENT CHALLENGES AND FUTURE PROSPECTS}

Despite many advantages of solid dispersion, issues related to preparation, reproducibility, formulation, scale up, and stability limited its use in commercial dosage forms for poorly water soluble drugs. Successful development of solid dispersion systems for preclinical, clinical, and commercial use has been feasible in recent years due to the availability of surface-active and selfemulsifying carriers with relatively low melting points. Because of the simplicity of manufacturing and scale up processes, the physicochemical properties and, as a result, the bioavailabilities of solid dispersions are not expected to change significantly during the scale up. For this reason, the popularity of the solid dispersion system to solve difficult bioavailability issues with respect to poorly water-soluble drugs will grow rapidly. One major focus of future research will be the identification of new surface-active and self-emulsifying carriers for solid dispersions. Only a small number of such carriers are currently available for oral use.

One limitation in the development of solid dispersion systems may the inadequate drug solubility in carriers, so a wider choice of carriers will increase the success of dosage form development. Research should also be directed toward identification of vehicles or excipients that would retard or prevent crystallization of drugs from supersaturated systems. Attention must also be given to any physiological and pharmacological effects of carriers used. Many of the surface-active and self-emulsifying carriers are lipidic in nature, so potential roles of such

\section{REFERENCES}

1. Venkat B,Yadav AV.Enhancement of solubility and dissolution rate of bcs class ii pharmaceuticals by nonaquious granulation technique. International journal of pharma. research and development2010;1 (12).

2. Remington: The Science and Practice of Pharmacy, 21st ed., D. B. Troy, Ed; Baltimore,Maryland, USA: Lippincott Williams \& Wilkins.

3. Patidar k. Solid Dispersion Technology: A Boon for Poor Water Soluble Drugs. Indian Journal of Novel Drug delivery2011;2 (3):83-90.

4. Nadia Saffoon RU.Enhancement of Oral Bioavailability and Solid Dispersion: A Review. Journal of Applied Pharmaceutical Science2011; 07 (01):13-20.

5. Vikas A SaharanVK. Dissolution Enhancement of Drugs. International Journal of Health Research2009;2 (2):107-124.

6. Mayerson M, Gibaldi M. New method of solid state dispersion for increasing dissolution rates. J Pharm Sci1966; 55: 1323-1342.

7. Shinde SS. An approach for solubility enhancement: solid dispersion. International Journal of Advances in Pharmaceutical Sciences2010;299-308.

8. Dhirendra K. Solid dispersion: A review. Pak. J. Pharm. Sci April 2009;22 (2):234-246.

9. Akiladevi D. Preparation and evaluation of paracetamol by solid dispersion technique. International Journal of Pharmacy and Pharmaceutical Sciences2011;3 (1):188-191.

10. Chiou WL,Riegelman S. Preparation and Dissolution Characteristics of Several Fast-Release Solid Dispersions of Griseofulvin.J Pharm Sci. 1969; 58(12): 1505-1510.

11. Jadhav AA. Improvement of Solubility and Dissolution Rate of Indomethacin by Solid Dispersion in Polyvinyl Pyrrolidone carriers on drug absorption, especially on their inhibitory effects on CYP3-based drug metabolism and $p$ glycoprotein-mediated drug efflux, will require careful consideration.

Physical and chemical stability of both the drug and the carrier in a solid dispersion are major developmental issues so future research needs to be directed to address various stability issues. Although, as mentioned earlier, the direct filling of solid dispersion into hard gelatin capsules is a relatively simple process, there are very limited reports on the scale up of the technology. Further studies on scale up and validation of the process will be essential. Many problems and challenges still remain with solid dispersion systems. Nevertheless, as a result of recent breakthroughs, it will continue to be one of the exciting frontiers of drug development.

\section{CONCLUSION}

Due to the advantageous features of solid dispersions formulation scientists consider it as one of the most potential method of improving oral bioavailability. But changes in crystal behavior of drug and/or carrier particles during processing and storage limits the method to be commercially applicable.Manufacturing of solid dispersions requires a perfect combination of drug to carrier(s). Carrier molecules play the most important role in enhancing solubility of the resultant dispersion and hence improvement in oral bioavailability. Whatever this technology is also highly potential to formulate controlled release dosage forms as the carriers may enhance or delay drug release.

K30 and Poloxomer 188. Asian J. Pharm. Tech2012; 2 (3):116-122.

12. Lalitha Y.Enhancement of dissolution of nifedipine by surface solid dispersion technique. International Journal of Pharmacy and Pharmaceutical Sciences 2011; 3:41-46.

13. Sanjay Kumar Das SR. Solid Dispersions : An Approach to Enhance the Bioavailability of poorly water soluble drugs. International Journal of Pharmacology and Pharmaceutical Technology2011;1 (1):2277 - 3436 .

14. Ruchi Tiwari GT. Solid Dispersions: An Overview To Modify Bioavailability Of Poorly Water Soluble Drugs. Int.J. PharmTech Res 2009;1 (4):1338-1349.

15. Tapan Kumar Giri AA. Physicochemical Classification and Formulation Development Of Solid Dispersion Of Poorly Water Soluble Drugs: An Updated Review. International Journal of Pharmaceutical \& Biological Archives2010;1 (4):309-324.

16. Anupama Kalia MP. Solid Dispersions: an approach towards enhancing dissolution rate. International Journal of Pharmacy and Pharmaceutical Sciences2011; 3 (4):9-19.

17. RahulM.Patil AH. Solid Dispersion: strategy to enhance solubility. International Journal of Pharmaceutical Sciences Review and Research2011;8 (2):66-73.

18. Ladan Akbarpour Nikghalb G S. Solid Dispersion: Methods and Polymers to increase the solubility of poorly soluble drugs. Journal of Applied Pharmaceutical Science2012; 2 (10): 170-175,

19. RenuKalyanwatSP. Solid Dispersion: a method for enhancing drug dissolution. International Journal of Drug Formulation \& Research 2010;1 (3);1-14. 
20. Mogal S A GP. Solid dispersion technique for improving solubility of some poorly soluble durgs. Der Pharmacia Lettre2012;4 (5):1574-1586.

21. Jatinder kaur GA. (2012). Improvement of drug solubility using solid dispersion. International Journal of Pharmacy and Pharmaceutical Sciences2012;4 (2);47-53.

22. Bhise SB, Rajkumar M. Effect of HPMC on Solubility and Dissolution of Carbamazepine Form III in Simulated Gastrointestinal Fluids. Asian Journal of Pharmaceutics 2008;2(1):38-42.

23. Santhosh Aruna MAK.Solid Dispersions - an approach to enhance the dissolution rate of irbesartan. international journal of research in pharmacy and chemistry2011; 1 (4):2231-2781.

24. Bhawana Kapoor R K.Solid dispersion: an evolutionary approach for solubility enhancement of poorly water soluble drugs. International Journal of Recent Advances in Pharmaceutical Research2012; 2 (2): 1-16.

25. Asker AF, Whitworth CW.Dissolution of acetylsalicylic acid from acetylsalicylic acid-polyethylene glycol 6000 coprecipitates. Pharmazie1975; 30:530-531.

26. Mogal SA, Gurjar P N, Yamgar DS, Kamod AC. Solid dispersion technique for improving solubility of some poorly soluble drugs.Der Pharmacia Lettre 2012; 4 (5):1574-1586.
27. Rakesh P,Patel D J. Physicochemical Characterization and Dissolution Study of Solid Dispersions of Furosemide with Polyethylene Glycol 6000 and Polyvinylpyrrolidone K30. Dissolution Technologies 2008; 17-25.

28. Puckhraj Chhaprel,Amit Talesara,Amit K Jain.Solubility enhancement of poorly water soluble drug using spray drying technique. International Journal of Pharmaceutical Studies and Research2012; Vol III/issue II :2229-4619.

29. KhanMS.Solid dispersions: Formulation, characterisation, permeability and genomic evaluation. Aston University.2010.

30. Dalwadi Sonali ST.Silymarin-solid dispersions: Characterization and influence of preparation methods on dissolution. Acta Pharm 2010; 427-443.

31. Joshi Vishalkumar H A. Development and evaluation of diclofenac sodium solid dispersion by mixed hydrotropic technique. International Journal of Pharmaceutical research and development2011;3 (8): 90-96.

32. Lakshmi NarasaiahV, Bhaskar Jimidi, Venkateswarlu Goli,Vijaya Bhaskar Kanakam. Enhancement of dissolution rate of atorvastatin calcium using solid dispersions by dropping method.International Journal of PharmTech Research 2011;CODEN (USA): IJPRIF ISSN : 0974-4304 Vol. 3, No.2, pp 652-659. 